\title{
Tratamiento quirúrgico de la bursitis nucal aséptica en equinos
}

\author{
Evaluation of surgical treatment of aseptic nuchal bursitis in horses
}

\author{
BUITRAGO M, JHONNY ${ }^{1}$ M.Sc, CARDONAA, JOSÉ ${ }^{*}$ Ph.D, MONTES V, DONICER ${ }^{2}$ Ph.D.
}

'Universidad de Córdoba, Facultad de Medicina Veterinaria y Zootecnia, Grupo de Investigación en Medicina de Grandes Animales (MEGA), Montería, Colombia. ${ }^{2}$ Universidad de Sucre, Facultad de Ciencias Agropecuarias, Departamento de Zootecnia, Grupo de Investigación en Mejoramiento y Reproducción Animal, Sincelejo, Colombia.

\section{Keywords:}

Hygromas;

fistulas:

abscess;

horses.

\section{Abstract}

An experimental study was performed by means of a clinical trial in seven horses to determine the effectiveness of surgical removal of the cranial nuchal bursa and / or caudal nuchal bursa under field conditions as a resolution therapy for aseptic nuchal bursitis. $5(71.5 \%)$ of the operated animals showed first intention scarring and 2 individuals $(28.5 \%)$ had wound dehiscence, and $5(71.5 \%)$ of the animals submitted to the surgical procedure took eight weeks in Achieve a full recovery. In all cases surgical removal of the nuchal bursa allowed the clinical recovery of the animals with aseptic nuchal bursitis in a period of eight to twelve weeks. Nuchal bursitis is a relatively unknown and relatively unknown pathology whose prevalence is unknown in Colombia, so it is necessary to develop more research in this pathology.

\section{Resumen}

Se realizó un estudio experimental mediante ensayo clínico en siete equinos para determinar la efectividad de la remoción quirúrgica de la bursa nucal craneal y/o la bursa nucal caudal en condiciones de campo como terapia de resolución de la bursitis nucal aséptica. 5 (71.5\%) de los animales intervenidos presentaron una cicatrización por primera intención y 2 ejemplares $(28,5 \%)$ presentaron dehiscencia de la herida, así mismo $5(71,5 \%)$ de los animales sometidos al procedimiento quirúrgico tardaron ocho semanas en alcanzar una recuperación total. En todos los casos la extirpación quirúrgica de la bursa nucal permitió la recuperación clínica de los animales con bursitis nucal aséptica en un periodo de ocho a doce semanas. La bursitis nucal es una patología poco estudiada y relativamente desconocida cuya prevalencia es desconocida en Colombia por lo que es necesario desarrollar más investigaciones en esta patología. 


\section{Introducción}

La bursa atlantal o nucal craneal se presenta sobre el arco dorsal del atlas ventral a la porción funicular del ligamento nucal, la bursa nucal caudal está localizada entre el proceso espinoso del axis y la porción funicular del ligamento nucal. Estas bursas se interponen entre estructuras sometidas a fricción o en puntos de presión inusual tal como prominencias óseas y tendones. La bursa atlantal es susceptible de inflamación produciendo una bursitis que es conocida como mal de la nuca,y de manera coloquial se le ha denominado muchas zonas de Colombia como "Aguacates" siendo una de las enfermedades más comunes de la parte craneal del cuello. Las bursitis de esta zona pueden ser de origen séptico, siendo ocasionadas por bacterias como Brucella abortus, Streptococcus spp y Staphylococcus spp. o por parásitos como Onchocerca cervicalis o más frecuentemente de origen traumático. También pueden clasificarse en agudas o crónicas, siendo en este último caso resultado de lesiones repetitivas, que se caracterizan por el cumulo de líquido bursal y el engrosamiento de las paredes de la bolsa por la formación de tejido fibroso. Los signos clínicos asociados con bursitis nucal craneal o caudal incluyen inflamación y dolor a la palpación, así como rigidez del cuello. En general las bursitis pueden ser manejadas de manera conservadora principalmente mediante el uso de antinflamatorios intra bursales, pero frecuentemente es necesario el desbridamiento quirúrgico para producir resolución de los signos clínicos.

Las bolsas sinoviales son sacos cerrados, aplanados, tapizados por una membrana celular de tejido conectivo, prácticamente idéntica a la membrana sinovial de las articulaciones, que se interpone entre estructuras sometidas a fricción (partes móviles) o en puntos de presión inusual tal como prominencias óseas y tendones (McILWRAITH, 2004; DYSON, 2011a); estas bursas contienen un líquido similar al sinovial pero difiere de este en la viscosidad y el coagulo de mucina, lo que sugiere diferencias en la cantidad o calidad del hialuronato que contienen (McILWRAITH, 2004), adicionalmente las bursas difieren de las bursas tendinosas en que no rodean por completo las estructuras tendinosas y están localizadas solo a un lado del tendón, frecuentemente interponiéndose entre él y una prominencia ósea (BAXTER, 2012).

En caballos estas bursas se clasifican según su origen o método de formación en congénitas y adquiridas, y según su localización anatómica en subcutáneas, subtendinosas, submusculares o subligamentosas (McILWRAITH, 2004; DYSON, 2011a; BAXTER, 2012); Las bursas congénitas con aquellas que hacen parte de las estructuras anatómicas normales y se desarrollan antes del nacimiento, se encuentran en ubicaciones constantes y a menudo asociadas a estructuras profundas como fascia, músculos y tendones; Las adquiridas se dan después del nacimiento , usualmente son subcutáneas y generalmente se producen como respuesta a trauma continuo por lo que también se han denominado como reactivas o funcionales (BAXTER, 2012).

Existen tres bursas asociadas al ligamento nucal, son la Bursa atlantal o nucal craneal (Bursa subligamentosa nuchalis cranialis), que se presenta sobre el arco dorsal del atlas ventral a la porción funicular del ligamento nucal, la Bursa nucal caudal (Bursa subligamentosa nuchalis caudalis) la cual está localizada entre el proceso espinoso del axis y la porción funicular del ligamento nucal y la Bursa supraespinosa (Bursa subligamentosa supraespinal) la cual está presente sobre los procesos más prominentes de la cruz (SISSON, 1979; DYSON, 2011b).

Las bursas subligamentosas son el tipo menos problemático de bursa y tiene pocas implicaciones clínicas, los ejemplos más notables son las bursas atlantal y la Bursa supra espinosa (BAXTER, 2012). La bursa atlantal o bursa nucal una vez se inflama produce una bursitis que es conocida como mal de la nuca (McILWRAITH, 2004; BAXTER, 2012), siendo una de las enfermedades más comunes de la parte craneal del cuello (GOLLOB et al., 2002). Esta denominación se da debido a que, al distenderse la Bursa, esta se extiende sobre la mitad de la superficie dorsal del atlas en una dirección cráneo caudal y lateralmente más allá de los límites del ligamento nucal (ABUJA et al., 2014), que puede ser observada como un aumento de tamaño localizado de tejido blando (DYSON, 2011a). 
Las bursitis se definen como una reacción inflamatoria dentro de una bolsa sinovial, que puede ir desde una inflamación leve hasta una bursitis séptica, aunque el trauma es la causa más común, y en general las bursitis no infecciosas son mucho menos sintomáticas que aquellos con bursitis infecciosas. Los aumentos de tamaño bursales aparecen como tumefacciones frías e indoloras y a menos que se agranden lo suficiente no interferirán con la función mecánica de la región donde se encuentran, la mayoría de los casos solo son problemas cosméticos a menos que se contaminen (McILWRAITH, 2004; BAXTER, 2012).

Las bursitis pueden darse de manera aguda o crónica, siendo en este último caso resultado de lesiones repetitivas que luego se vuelven clínicamente inaceptables, que se caracterizan por el cumulo de líquido bursal y el engrosamiento de las paredes de la bolsa por la formación de tejido fibroso. Dentro de la cavidad bursal se pueden desarrollar bandas fibrosas o septos y con frecuencia se produce un engrosamiento subcutáneo generalizado alrededor de la Bursa. (McILWRAITH, 2004).

En las bursitis sépticas los signos clínicos pueden ser abruptos o insidiosos, frecuentemente existe dolor, rigidez de cuello y pueden presentarse con tractos fistulosos o como una inflamación difusa sin drenaje. (MCILWRAITH, 2004; MAIR \& DIVERS, 2009; BAXTER, 2012). Este tipo de bursitis son causadas frecuentemente por bacterias como Brucella abortus, Streptococcus spp y Staphylococcus spp. (HAWKINS \& FESSLER, 2000; MAIR \& DIVERS, 2009; GARCIA et al., 2010) o por parásitos como Onchocerca cervicalis (HAWKINS \& FESSLER, 2000).

En caballos con dolor cervical se debe considerar siempre como diagnóstico diferencial la bursitis nucal craneal y caudal (GARCIA et al., 2010), por que causa frecuentemente una postura cervical anormal (DYSON, 2011a,b). Los signos clínicos asociados con bursitis nucal craneal o caudal incluyen inflamación y dolor a la palpación, pero estos pueden pasar desapercibidos en caballos con afecciones moderadas a leves (GARCIA et al., 2010), el diagnostico depende usualmente de la identificación ultrasonografica de una Bursa distendida dorsal a la primera y segunda vértebra cervical (DYSON, 2011b).
En general las bursitis subcutáneas pequeñas y las subligamentosas agudas responden bien al tratamiento conservador y tienen un buen pronóstico. Las bursitis más crónicas y las bursitis infectadas pueden ser un gran reto clínico siendo su pronóstico un poco más reservado (BAXTER, 2012) siendo frecuentemente necesario el desbridamiento quirúrgico para producir resolución de los signos clínicos (HAWKINS \& FESSLER, 2000; DYSON, 2011a).

\section{Materiales y métodos}

Sitio de estudio. El estudio fue realizado en el departamento de Córdoba, ubicado entre las coordenadas $7^{\circ} 23^{\prime}$ y $9^{\circ} 26^{\prime} \mathrm{LN}$ y los $74^{\circ} 52^{\prime}$ y $76^{\circ}$ $32^{\prime}$ LO, a una altura de $30 \mathrm{msnm}$, con temperatura promedio anual de $28{ }^{\circ} \mathrm{C}$, humedad relativa del $82 \%$, precipitación media anual de $1400 \mathrm{~mm}$ y pertenece a la formación climática de bosque tropical lluvioso. Se presentan dos estaciones bien definidas (época de lluvia y época seca) (PABON et al., 2001).

Se realizó un estudio experimental mediante ensayo clínico para determinar la efectividad de la remoción quirúrgica de la Bursa nucal craneal y/o la Bursa nucal caudal en condiciones de campo como terapia de resolución de la bursitis nucal aséptica, para lo cual se realizó la cirugía a siete equinos de distintos sexos y edades que padecían la enfermedad y a los cuales se les descarto el origen séptico de la alteración mediante la realización de hemogramas, análisis del líquido bursal y serología para descartar infecciones bacterianas, infección por Brucella abortus, así como la presencia de microfilarias.

Procedimiento quirúrgico. Lo animales fueron sedados con Xilacina al 10\% (Anased $($ ) a dosis de $1 \mathrm{mg} / \mathrm{Kg}$, posteriormente se realizó tricotomía de la región atlanto occipital, en un área aproximada de $15 \times 10 \mathrm{~cm}^{2}$, una vez depilado los animales fueron anestesiados usando una mezcla de Xilacina al 10\% (Anased $\AA$ ) a dosis de $0,5 \mathrm{mg} / \mathrm{Kg}$, Ketamina al 10\% (Imalgene 1000®) a dosis de $3 \mathrm{mg} / \mathrm{Kg}$ y diazepam a dosis de 0,1 $\mathrm{mg} / \mathrm{Kg}$ preparado en la misma jeringa y aplicado vía intra venosa en bolo de infusión. Una vez el animal estuvo en recumbencia lateral se realizó lavado quirúrgico con yodopovidona y alcohol (embrocado quirúrgico). Una vez preparada el 
área se realizó una incisión sobre la piel con una longitud de 9 a $15 \mathrm{~cm}$, posteriormente se realizó disección roma de la fascia subcutánea, musculo subcutáneo y el ligamento nucal, ubicando la bursa afectada mediante palpación. Una vez delimitada se desbridó el tejido adyacente y se retiró en su totalidad respetando siempre la integridad del ligamento nucal. En todos los casos el tejido cercano a la bursa nucal presento grados variables de necrosis y tejido denso que fue extraído durante la desbridación.

Manejo postquirúrgico. Para el manejo postoperatorio fue instaurado un tratamiento antibiótico con penicilina benzatinica a dosis de $22.000 \mathrm{U}$. I/Kg vía intramuscular, se recomendó el lavado de la herida quirúrgica diariamente con agua hervida y jabón, así como la aplicación de un cicatrizante tópico (Pezosan $\mathrm{N}^{\circledR}$ ) hasta el cierre completo de la herida.

Para el manejo del dolor y la inflamación se utilizó flunixin meglumine a dosis de $1,1 \mathrm{mg} / \mathrm{Kg}$ intravenoso cada 24 horas por 3 días, así como una dilución de sulfato de magnesio que se aplicaba de manera tópica durante quince minutos dos veces al día por cinco días. Todos los animales fueron retirados de sus labores por un espacio de tres semanas.

Durante el posquirúrgico se evaluó el tiempo total de cicatrización, la presencia de inflamación y secreciones, así como el aumento de tamaño del área atlanto occipital. Para evaluar la inflamación se determinó el grado de dolor máximo en cada uno de los animales, el cual fue determinado por medio de la reacción a la palpación del área afectada y se clasifico en tres grados, siendo el grado 0 aquellos animales sin dolor que no reaccionaron a la manipulación, grado 1 animales con dolor leve a moderado que presentaban un rechazo sutil a la manipulación y grado 2 animales con dolor intenso que presentaron una reacción fuerte ante la manipulación. También se determinó el tiempo de duración del edema después de la cirugía.

\section{Resultados y Discusión}

En el posquirúrgico 5 (71.5\%) de los animales intervenidos presentaron una cicatrización por primera intención sin ningún tipo de complicaciones, y 2 ejemplares $(28,5 \%)$ presentaron dehiscencia de la herida y se realizó cicatrización por segunda intención debido a mal manejo de la herida por parte de los encargados. En la figura 1 y 2 se observa que el $5(71,5 \%)$ de los animales sometidos al procedimiento quirúrgico presentaron dolor y edema por ocho semanas después del procedimiento, y solo $2(28,5 \%)$ persistieron con dolor y edema por más de ocho semanas. Es notoria la correlación entre la presencia de edema y la reacción al dolor entre los animales intervenidos.

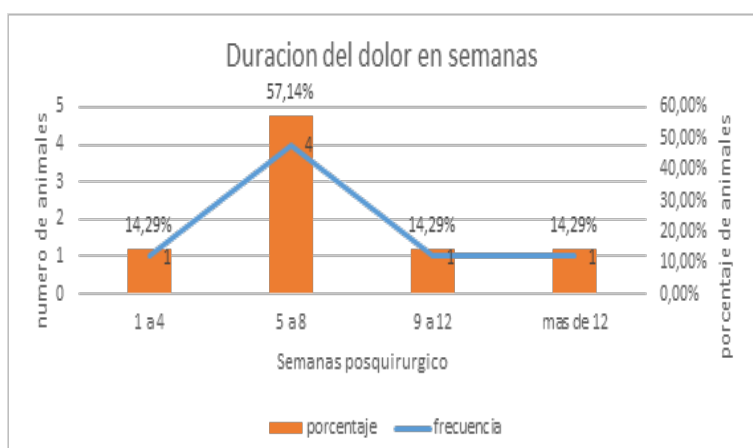

Figura 1. duración del dolor posquirúrgico en semanas de animales sometidos a bursectomia nucal craneal o caudal.

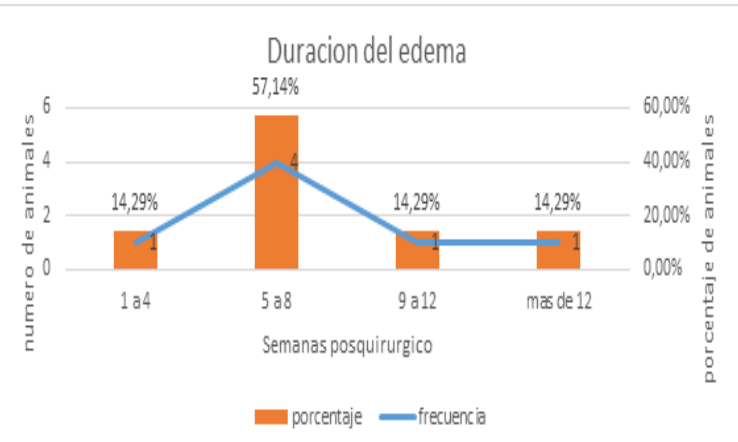

Figura 2. duración del edema posquirúrgico en semanas de animales sometidos a bursectomia nucal craneal o caudal.

En la figura 3 se observa que en general la cirugía produce en la mayoría de los casos $(85,6 \%)$ un dolor leve a moderado y solo un pequeño porcentaje $(14,2 \%)$ sufrió un dolor severo después de la intervención. 


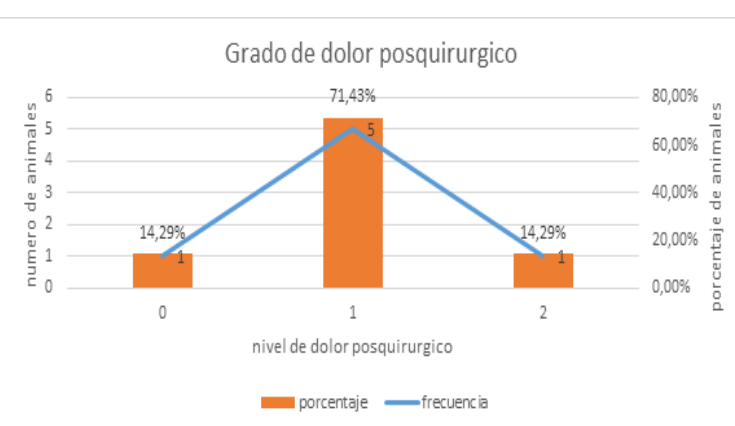

Figura 3. clasificación del dolor posquirúrgico en los animales sometidos a bursectomia nucal craneal y/o caudal.

En la figura 4 se muestra que el $57,1 \%$ de los animales duraron con un aumento de tamaño en la región atlanto occipital por un periodo de hasta ocho semanas, el $28,5 \%$ tardo hasta doce semanas, y solo un caso $(14,3 \%)$ tardo más de doce semanas.

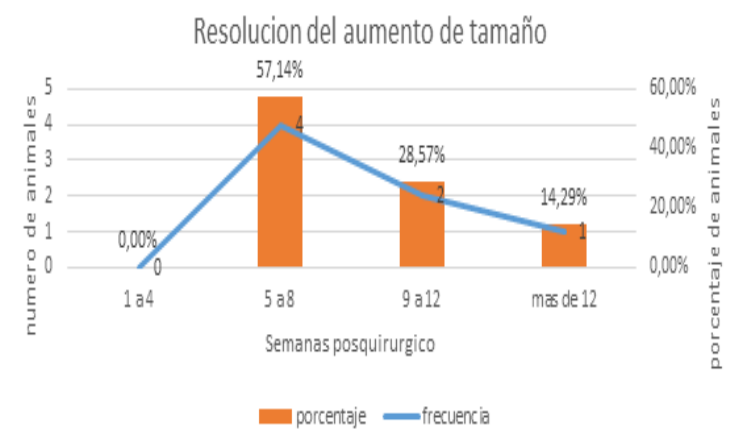

Figura 4: tiempo de resolución del aumento de tamaño en los animales sometidos a bursectomia nucal craneal y/o caudal.

La bursa nucal craneal es una estructura bilobulada localizada ventrolateralmente al ligamento nucal y dividida por un septum incompleto compuesto por una estructura similar a la sinovia, una cantidad variable de tejido graso y haces de fibras de la parte laminar del ligamento nucal. Cuando no está distendida descansa sobre el tercio medio y caudal de la superficie del atlas sobre su arco dorsal y se extiende caudalmente sobre la articulación entre $C 1$ y C2, contiene una pequeña cantidad de líquido sinovial, cuando se distiende completamente puede alcanzar un volumen de $60 \mathrm{ml}$, siendo la condición más común que afecta esta región es la bursitis nucal, pudiendo afectar el desempeño del atleta equino (ABUJA et al., 2014).

En estados unidos la bursitis nucal caudal o craneal es denominada "poll evil" y en este país es considerada una enfermedad poco común que afecta la poción funicular del ligamento nucal en su paso sobre el aspecto dorsal del atlas y el axis para insertarse en el hueso occipital. El examen ultrasonografico revela distención por fluidos y engrosamiento sinovial de la Bursa nucal craneal o caudal y la aspiración del fluido bursal permite cultivarlo para descartar etiologías infecciosas como Brucella spp. La radiografía puede revelar remodelación ósea y mineralización o radiolucidés del aspecto dorsal del atlas o axis (HAUSSLER, 2016).

Algunos autores consideran que la bursitis nucal es una patología secundaria del ligamento nucal a nivel de la primera y segunda vértebra cervical (BAXTER, 2012). La respuesta al manejo medico con anti inflamatorios y ácido hialuronico intra bursales es limitada (GARCIA et al., 2010) y se ha descrito en caballos y mulas secundaria a trauma directo y a la introducción de infecciones dentro de una Bursa previamente traumatizada. Las bacterias asociadas a la bursitis atlantal incluyen Brucella abortus, Streptococcus zooepidemicus y Actinomices bovis, este último particularmente en animales que conviven junto con bovinos (BENDREY et al., 2009).

El diagnostico de bursitis depende de la Bursa especifica que este afectada y la causa inicial (BAXTER, 2012), particularmente la bursitis nucal es un diagnostico difícil de confirmar basado solo en los signos clínicos y la ultrasonografía (BAXTER, 2012) ya que hay pocos reportes de imágenes diagnósticas y técnicas endoscópicas (ABUJA et al., 2014), sin embargo se ha descrito que la mayoría de los problemas bursales pueden ser diagnosticados con una combinación de radiología (incluyendo radiología de contraste) y ultrasonografía (BAXTER, 2012).

La ecografía presenta la ventaja de proveer un diagnóstico más preciso debido a que esta modalidad de imagen permite la valoración de los tejidos blandos asociados a una Bursa nucal específica, así como la superficie del proceso espinoso vertebral subyacente, la 
distención por fluidos y el engrosamiento sinovial; adicionalmente en ocasiones puede ser útil para distinguir los procesos sépticos de los asépticos antes de la evaluación citológica y el cultivo bacteriano de las muestras, aunque en algunas situaciones esto puede ser difícil, especialmente en condiciones de cronicidad (GARCIA et al., 2010; HAUSSLER, 2016). Es necesario mencionar que se ha reportado que en ocasiones no es posible observar la bursa atlantal mediante ecografía (GOLLOB et al., 2002). Cuando el proceso patológico asociado a la Bursa nucal craneal o caudal se extiende y ocasiona inflamación del hueso subyacente puede ser útil la técnica radiográfica, además permite evaluar la extensión de la distensión de la Bursa y el daño óseo asociado revelando remodelaciones óseas y mineralización o radiolucides del aspecto dorsal del atlas o axis (MAIR \& DIVERS, 2009; GARCIA et al., 2010; HAUSSLER, 2016). El examen ultrasonográfico y la resonancia magnética son técnicas que pueden ser usadas en los casos clínicos para caracterizar la bursitis nucal más que para ayudar en la planeación pre quirúrgica (ABUJA et al., 2014). Se puede realizar aspiración del fluido bursal para cultivo si se sospecha de etiologías infecciosas como Brucella spp. (HAUSSLER, 2016).

El tratamiento de las bursitis varía considerablemente dependiendo de la localización, duración y presencia de infección u otros problemas musculo esqueléticos primarios, en general se recomienda iniciar con tratamientos conservadores a menos que haya presencia de infección (BAXTER, 2012). Los tratamientos conservadores descritos para la bursitis nucal aséptica incluyen el uso combinado de antiinflamatorios tópicos y sistémicos junto con vendajes de presión, así como la inyección eco guiada de corticoides y ácido hialuronico intra bursales. En las afecciones más crónicas se recomienda el manejo mediante drenaje y administración de corticoides o compuestos yodados. Estos tratamientos pueden presentar una respuesta limitada, requiriendo la debridacion quirúrgica (GARCIA et al., 2010; BAXTER, 2012).

El manejo de las bursitis mediante debridacion por apertura quirúrgica con el caballo en pie tiene como ventaja que permite observar las estructuras afectadas y accesar quirúrgicamente para la debridacion, sin embargo, requiere un manejo frecuente de la herida en el posquirúrgico (GARCIA et al., 2010).

Todos los animales de este estudio mostraron resolución completa de los signos clínicos tal como lo reportan otros autores (GARCIA et al., 2010; BAXTER, 2012) y pudieron regresar a sus labores entre las ocho y doce semanas después de la cirugía, siendo una opción para el manejo de las bursitis crónicas en campo. Se han encontrado pocos reportes de literatura acerca del manejo de las bursitis nucales en equinos tanto de forma medica como quirúrgica, uno de los pocos reportes fue realizado en estados unidos en donde se describió la desbridación mediante bursoscopia nucal como una técnica alternativa a la intervención quirúrgica, con la cual se ha obtuvo una alta tasa de éxito y una recuperación más rápida de los pacientes (GARCIA et al., 2010), sin embargo presenta como desventaja el alto costo de los equipos y la necesidad de personal especializado en su manejo, así como la dificultad de realizarla en condiciones de campo.

Este es el primer reporte en Colombia del manejo quirúrgico de la bursitis nucal en equinos. Se desconoce la prevalencia de esta enfermedad en el medio y su impacto en la salud y el desempeño de los animales afectados, así como sus implicaciones desde el punto de vista económico, por lo que es necesario realizar más investigaciones que permitan determinar la importancia de esta patología en las distintas regiones del país.

\section{Conclusiones}

La extirpación quirúrgica de la bursa nucal permite la recuperación clínica de los animales con bursitis nucal aséptica en un periodo de ocho a doce semanas si se garantizan los cuidados adecuados de la herida quirúrgica, permitiendo la resolución de la sintomatología asociada a la bursitis nucal.

La bursitis nucal es una patología poco estudiada y relativamente desconocida por ser de poca presentación en países desarrollados, pero cuya prevalencia es desconocida en Colombia por lo que es necesario ahondar en el conocimiento de su epidemiologia en las condiciones locales para determinar su impacto en el estatus sanitario de los equinos en Colombia. 


\section{Referencias}

ABUJA, G., GARCIA LOPEZ, J., MANSO DIAZ, G., SPOORMAKERS, T., \& TAEYMANS, O. 2014. The cranial nuchal bursa: Anatomy, ultrasonography, magnetic resonance imaging and endoscopic approach. Equine Veterinary Journal, 46, 745-750.

BAXTER, G. 2012. Management of bursitis. En J. Auer, \& J. Stick, equine surgery. 4 ed., págs. 1148-1157.

BENDREY, R., CASSIDY, J., BKOVENKO, N., LEPETZ, S., \& ZAITSEVA, G. 2009. A Possible Case of 'Poll-Evil' in an Early Scythian Horse Skull from Arzhan 1, Tuva Republic, Central Asia. International Journal of Osteoarchaeology, 111-118.

DYSON, S. 2011a. Bursae and other soft tissue swellings. En M. Ross, \& S. Dyson, Diagnosis and Management of Lameness in the Horse. 2 ed., págs. 799-802.

DYSON, S. 2011b. Lesions of the equine neck resulting in lameness or poor performance. Vet Clin Equine, 27, 417-437.

GARCIA LOPEZ, J., JENEI, T., CHOPE, K., \& BUBECK, K. 2010. Diagnosis and management of cranial and caudal nuchal bursitis in four horses. J Am Vet Med Assoc, 237(7), 823-829.

GOLLOB, E., EDINGER, H., STANEK, C., \& WURNIG, C. 2002. Ultrasonographic investigation of the atlanto-occipital articulation in the horse. Equine Veterinary Journal, 34(1), 44-50.

HAUSSLER, K. 2016. Spinal Pathology of the Cervical and Cervicothoracic Regions. AAEP $360^{\circ}$ Pain in the neck, págs. 1-7.

HAWKINS, J., \& FESSLER, J. 2000. Treatment of supraspinous bursitis by use of debridement in standing horses: 10 cases (1968-1999). J Am Vet Med Assoc, 217, 74-78.

MAIR, T., \& DIVERS, T. 2009. Brucellosis In The Horse. En E. V.-P.-R. Book, Infectious Diseases of the Horse. págs. 275-280.

MCILWRAITH, C. 2004. Enfermedades de las bursas y otras estructuras periarticulares. En Stashak, T.S, \& D. Troy (Ed.), ADAMS claudicaciones en equinos. Buenos Aires: Inter medica. págs. 680-684.

PABON, J., ESLAVA, J., \& GOMEZ, R. 2001. Generalidades de la distribucion espacial y temporal de la temperatura del aire y de la precipitacion en Colombia. Meteorologia colombiana, 4: 47-59.

SISSON, S. 1979. Sindesmologia de los equinos. En S. Sisson, J. Grossman, \& R. Getty (Ed.), Anatomia de los animales domesticos. Elsevier masson. 5 ed., págs. 392-420. 